\title{
CNS LYMPHOMA MIMICKING A DEMYELINATING LESION
}

\author{
Akkamahadevi V. Nipanal ${ }^{1}$, R. Madhumathi², Navya $^{3}$, Angel ${ }^{4}$
}

\section{HOW TO CITE THIS ARTICLE:}

Akkamahadevi V. Nipanal, R. Madhumathi, Navya, Angel "CNS Lymphoma Mimicking a Demyelinating Lesion". Journal of Evolution of Medical and Dental Sciences 2015; Vol. 4, Issue 66, August 17; Page: 11555-11559, DOI: $10.14260 /$ jemds/2015/1666

ABSTRACT: CNS presentation of Diffuse Large B-Cell Lymphoma (DLBCL) is reported more frequently as secondary to systemic primary. The incidence of CNS infiltration of DLBCL varies from 1.1 to $10.4 \%$. The main risk factor for infiltration of the CNS by lymphoma is the histological subtype, with much higher rates for Burkitt's and T-lymphoblastic lymphomas. We present a case of DLBCL with relapsing remitting illness with stepwise progression has not been previously described. DLBCL is an aggressive form of NHL and usually occurs in lymph nodes. Extranodal presentation, when it occurs most commonly involves the gastrointestinal tract. As our patient presented with CNS lymphoma with no other lesions in the body we considered the possibility of a primary CNS presentation, which is again a less common presentation. This case is also significant because the incidence of DLBCL, NHL has been noted to be increasing over the years. ${ }^{1}$ we conclude that DLBCL, when it occurs as a primary CNS lymphoma, has a presentation that can mimic a demyelinating lesion. Hence an early tissue diagnosis should be attempted in cases of suspected CNS lymphoma in view of the poor survival of these patients when appropriate treatment is not initiated in time.

KEYWORDS: DLBCL-Diffuse Large B-Cell Lymphoma. NHL-Non Hodgkins Lymphoma.

CASE REPORT: A 54 years old male presented with 2 months history of a relapsing remitting illness. The patient developed progressive left sided hemiparesis and acute confused state of few days duration following which he had an episode of seizures. He had associated symptoms of raised intracranial tension headache, nausea and vomiting. He complained of blurring of vision in both the eyes. He was evaluated briefly in a local hospital where his vision and sensorium improved over a few days with symptomatic treatment.

He had residual left hemiparesis. His medical condition remained unchanged for the next $1 \frac{1 / 2}{2}$ months. He subsequently developed right sided hemiplegia and lapsed into altered sensorium when he was referred to our hospital. He was admitted and his sensorium improved over the next few days. On examination he had motor aphasia, bilateral horizontal gaze nystagmus, right UMN facial palsy, motor weakness of both upper and lower limbs right>left, hypotonia and extensor plantar on the right.

Routine biochemistry and hematology was normal. S ACE level was $28 \mathrm{U} / \mathrm{L}$, S. Total Protein was $6.1 \mathrm{~g} / \mathrm{dl}$ and S. Albumin was 3.8g/dl. ELISA for HIV was negative. MRI Brain revealed multiple illdefined $\mathrm{T}_{2}$ and FLAIR hyper-intense lesions in the right frontal and parietal sub-cortical white matter, left centrum semiovale and right callososeptal interface (Fig. 2). The lesion was isointense on $\mathrm{T}_{1}$. Evidence of hemorrhage into the lesion was present as $\mathrm{T}_{1}$ sequence, blooming on SWI and mild diffusion restriction. No hyperintensitiy on CBV perfusion. On contrast- multiple disc enhancing and ring lesions present.

Open ring enhancing lesion seen in Right frontal lobe (Fig. 3). MRS showed a small lipid lactate peak but no increase in choline and no reduction in NAA. CSF revealed 2 cells, no malignant cells, CSF Glucose was 72gm/dl and CSF Protein was $136 \mathrm{gm} / \mathrm{dl}$. 
EEG revealed spike and wave and sharp and wave in T2- T4, T4- T6, F8- T4. Background showed diffuse slowing predominantly in fronto- temporal and alpha activity in parieto- occipital region. Chest and Abdominal imaging was normal.

Based on the imaging the differentials considered were demyelination, granulomatous disease, glioma, lymphoma. Demyelinating lesions are predominantly confined to the CNS white matter; acute demyelinating lesions enhance on contrast and have peri-lesional oedema. Oligoclonal bands were however absent in CSF although the CSF Proteins were elevated. Being in an area with a high prevalence of tuberculosis, ring or disk enhancing lesions could represent an infectious granulomatous lesion.

This was considered despite the relatively low CSF protein as against what is usually observed in TB meningo-encephalitis. Non-infectious granulomatous lesions including neurosarcoidosis was excluded with normal S. ACE level and normal Chest CT. Primary CNS tumours including lymphoma was considered in view of multiple ring and disc enhancing lesions with areas of haemorrhage within the lesion. The patient started on treatment for treatable causes with systemic steroids, anti-tubercular treatment, broad spectrum antibiotics, anti-oedema measures and antiepileptics.

The patient however continued to worsen and developed additional lesions on repeat CT Brain despite being on the above treatment. Hence it was decided that tissue diagnosis was necessary and stereotactic brain biopsy was done as there was no lesions else ware in the body.

Stereotactic biopsy was done for tissue diagnosis. White matter revealed infiltration by large neoplastic lymphoid cells with vesicular nuclei, prominent eosinophilic nucleoli, diffusely distributed (Fig 1). Angiocentric pattern of distribution surrounding blood vessels, splitting vessel wall was also noted. Foamy histiocytes, mature lymphocytes and reactive astrocytes with tissue edema and breakdown was also noted. Immunohistochemistry showed that the tumor cells strongly expressed CD20 (B cell marker) and high LCA (CD45) MIB-1 labelling. Angiocentric distribution was highlighted by CD20 stain. CD3 T cells and CD68 histiocytes were also noted. Based on the histology a diagnosis of Non-Hodgkin's lymphoma (NHL), diffuse large B cell type (DLBCL) was made.

DISCUSSION: We present a case of DLBCL with an interesting clinical presentation. The presentation as a relapsing remitting illness with stepwise progression has not been previously described. DLBCL is an aggressive form of NHL and usually occurs in lymph nodes. Extranodal presentation, when it occurs most commonly involves the gastrointestinal tract. As our patient presented with CNS lymphoma with no other lesions in the body we considered the possibility of a primary CNS presentation, which is again a less common presentation. This case is also significant because the incidence of DLBCL, NHL has been noted to be increasing over the years. ${ }^{1}$ With the exception of skin malignancies, such temporal increases in cancer incidence are unprecedented.

DLBCL is the most common form of NHL. There is a male predominance with median patient age of 60 years in the cases described. DLBCL typically presents as a rapidly enlarging mass at a nodal or extranodal site. Extranodal sites include the gastrointestinal tract, skin, bone and brain. Bone marrow involvement is uncommon and late. The morphology of DLBCL features relatively large cell size, vacuolated nucleus, 2-3 prominent nucleoli and abundant cytoplasm. These cells are diffusely distributed thus earning them the name diffuse large B cell. The immunophenotype shows CD19, CD20 (Mature B cell markers) and CD10, BCL6 (Germinal center B cell markers).1,2 
CNS presentation of DLBCL is reported more frequently as secondary to systemic primary. The incidence of CNS infiltration of DLBCL varies from 1.1 to $10.4 \%$. The main risk factor for infiltration of the CNS by lymphoma is the histological subtype, with much higher rates for Burkitt's and T-lymphoblastic lymphomas. Survival after CNS infiltration was 1.9 months on average. ${ }^{3}$

Relapsing remitting disease course in this patient was possible due to the resolution of perilesional oedema with anti-oedema measures, in this case Syp Oral Glycerol. The relapsing remitting presentation typically brings up the possibility of demyelination. Demyeliation can have a very similar clinical and radiological presentation to lymphoma. Previous studies have made similar observations. Katu Y et al has described a case with neuropsychiatric presentation whose initial stereotactic biopsy showed demyelination on histopathology. The diagnosis of DLBCL was confirmed only on autopsy. 4

Diffuse Large B-Cell Lymphoma (DLBCL) remains a curable lymphoma, hence the need for aggressive evaluation to establish a diagnosis. ${ }^{1}$ the improved outcome is attributed to incorporation of rituximab in standard CHOP chemotherapy regimen. ${ }^{1}$

CONCLUSION: DLBCL, when it occurs as a primary CNS lymphoma, has a presentation that can mimic a demyelinating lesion. Hence an early tissue diagnosis should be attempted in cases of suspected CNS lymphoma in view of the poor survival of these patients when appropriate treatment is not initiated in time.

Fig. 1A \& 1B: Histopathology of stereotactic biopsy specimen - White matter revealed infilteration by large neoplastic Lymphoid cells with vesicular nuclei, prominent eosinophilic nucleoli diffusely distributed.

Fig. 1C: Tumour cells expressing CD20 (B cell marker).

Fig. 1D: High LCA (CD45) MIB-1 Labelling.

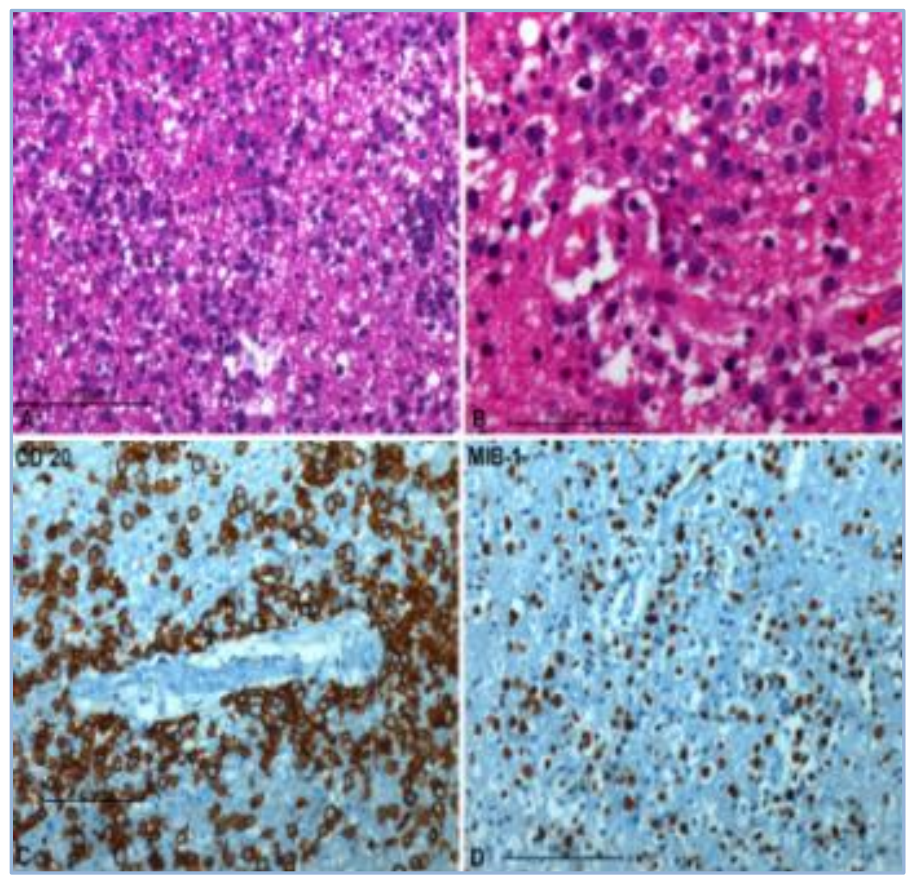

Fig. 1A, 1B, 1C \& 1D 


\section{CASE REPORT}

Fig. 2: MRI Brain - Multiple ill-defined $\mathrm{T}_{2}$ and FLAIR hyper-intense lesions in the right frontal and parietal sub-cortical white matter, left centrum semiovale and right callososeptal interface.

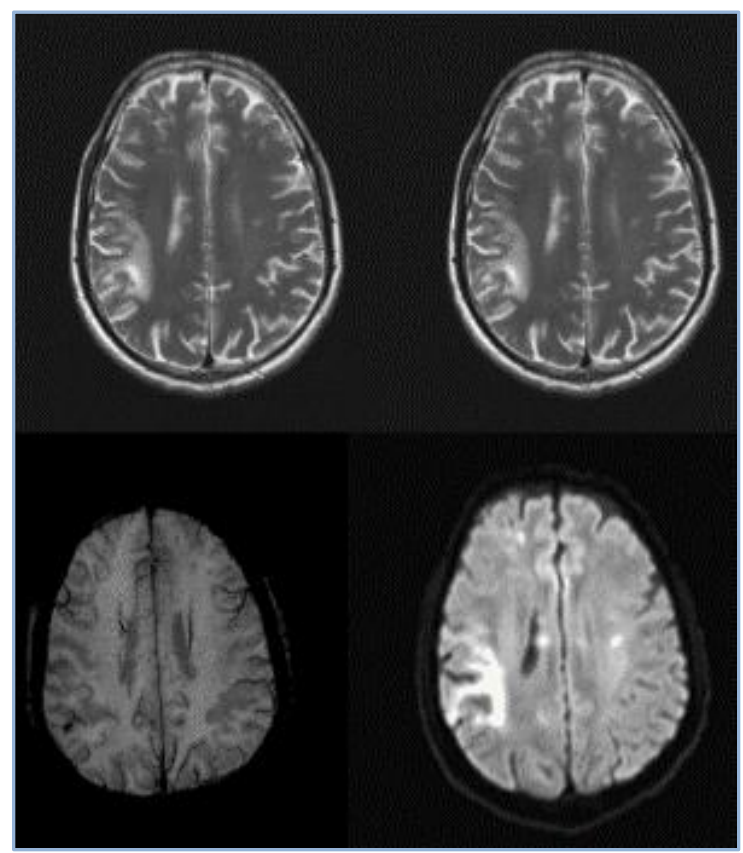

Figure 2

Fig. 3: MRI contrast- Multiple ring enhancing lesions present. Open ring enhancing lesion seen in Right frontal lobe.

MRS - A small lipid lactate peak.

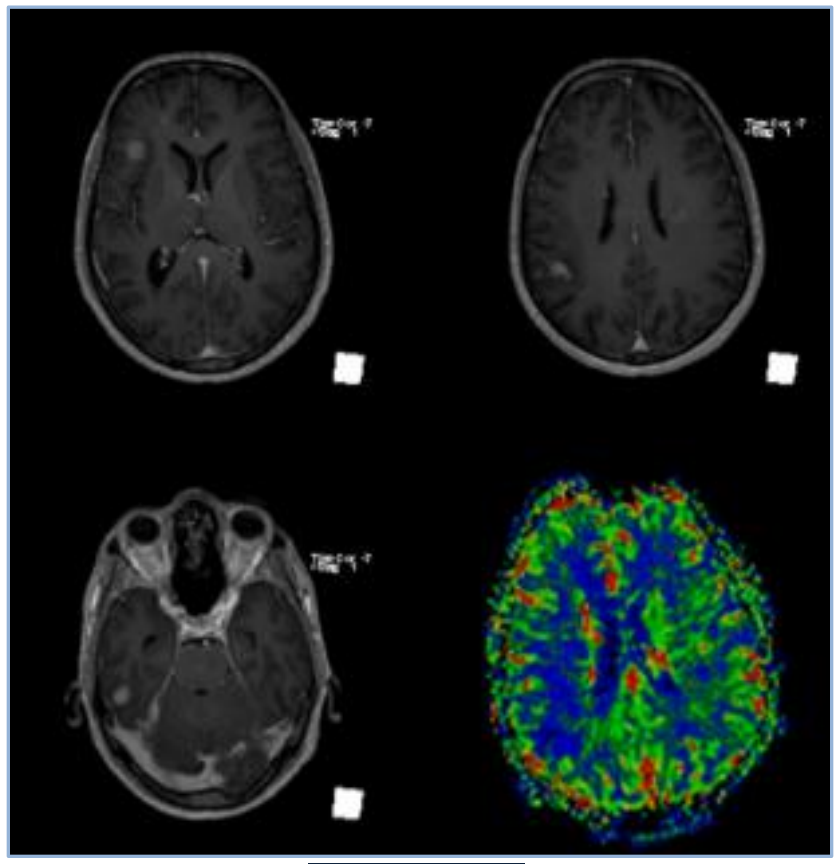

Figure 3 


\section{REFERENCES:}

1. Friedberg JW; Diffuse Large B Cell Lymphoma; Hematol Oncol Clin North Am. 2008 October; 22(5): 941-ix.

2. Kumar V, Abbas AK, Fausto N, Aster J; Robbins \& Cotran Pathologic Basis of Disease, Saunders, Elsevier Health Sciences, Eighth Edition; 589-620.

3. Bueno da Silveira da Rocha TM, Fortier SC, Pinto MSG, et al; Secondary infiltration of the central nervous system in patients with diffuse large B-cell lymphoma; Rev Bras Hematol Hemoter. 2013; 35(4):256-62.

4. Kato Y, Hayashi T, Kawai-Masaoka A, et al; Primary Central Nervous System Cytotoxic T-cell Lymphoma Mimicking Demyelinating Disease; Intern Med 53: 1197-1200.

\section{AUTHORS:}

1. Akkamahadevi V. Nipanal

2. R. Madhumathi

3. Navya

4. Angel

\section{PARTICULARS OF CONTRIBUTORS:}

1. Post Graduate Student, Department of General Medicine, Bangalore Medical College and Research Institute, Bangalore.

2. Associate Professor, Department of General Medicine, Bangalore Medical College and Research Institute, Bangalore.

3. Post Graduate Student, Department of General Medicine, Bangalore Medical College and Research Institute, Bangalore.

\section{FINANCIAL OR OTHER} COMPETING INTERESTS: None
4. Post Graduate Student, Department of General Medicine, Bangalore Medical College and Research Institute, Bangalore.

\section{NAME ADDRESS EMAIL ID OF THE CORRESPONDING AUTHOR:}

Dr. Akkamahadevi V. Nipanal,

Post Graduate Student, Department of General Medicine, Room No: 306, SVBL Vishranthi Dhama, Victoria Hospital Campus, Bangalore-560002, Karnataka.

E-mail: akkamahadevi77777@rediffmail.com

Date of Submission: 21/07/2015. Date of Peer Review: 22/07/2015. Date of Acceptance: 10/08/2015. Date of Publishing: 17/08/2015. 